\title{
TRUNCATED TETRAHEDRA AND THEIR REFLECTION GROUPS
}

\author{
T. H. MARSHALL \\ (Received 16 October 1995; revised 4 July 1997) \\ Communicated by J. R. J. Groves
}

\begin{abstract}
We outline the classification, up to isometry, of all tetrahedra in hyperbolic space with one or more vertices truncated, for which the dihedral angles along the edges formed by the truncations are all $\pi / 2$, and those remaining are all submultiples of $\pi$. We show how to find the volumes of these polyhedra, and find presentations and small generating sets for the orientation-preserving subgroups of their reflection groups.

For particular families of these groups, we find low index torsion free subgroups, and construct associated manifolds and manifolds with boundary. In particular, for each $g \geq 2$, we find a sequence of hyperbolic manifolds with totally geodesic boundary of genus $g$, which we conjecture to be of least volume among such manifolds.
\end{abstract}

1991 Mathematics subject classification (Amer. Math. Soc.): primary 30F40, 57N10; secondary $20 \mathrm{~F} 55$. Keywords and phrases: Coxeter polytope, truncated tetrahedron, co-volume, torsion-free subgroup, hyperbolic manifold.

\section{Introduction}

A Coxeter polytope is a polytope whose dihedral angles are all submultiples of $\pi$. The main interest of these polytopes is that their reflection groups (that is, the groups generated by reflections through their faces) are discrete. Coxeter himself [4] classified these polytopes in spherical and Euclidean space, showing that they must be, respectively, simplices, and Cartesian products of simplices. In hyperbolic space the situation is more complicated, and we consider only the 3-dimensional case. The situation for tetrahedra is well known, there being, up to isomorphism, exactly 32 Coxeter tetrahedra in hyperbolic 3-space, including those with one or more ideal vertices [7]. For other combinatorial types, the Coxeter polyhedra can be identified using Andreev's theorem [1].

(C) 1998 Australian Mathematical Society 0263-6115/98 \$A2.00+0.00 
The subject of this paper is the class of truncated tetrahedra which are also Coxeter polyhedra, where we define a truncated tetrahedron to be a polyhedron which is combinatorially equivalent to a tetrahedron with $k(1 \leq k \leq 4)$ of its vertices 'cut off' perpendicularly, in the obvious way. An example, with $k=2$, is illustrated in Figure 1. For $k=1$ a truncated tetrahedron is a triangular prism. These polyhedra and their reflection groups have been discussed by Conder and Martin [3]. In this paper we extend and develop some of their ideas.

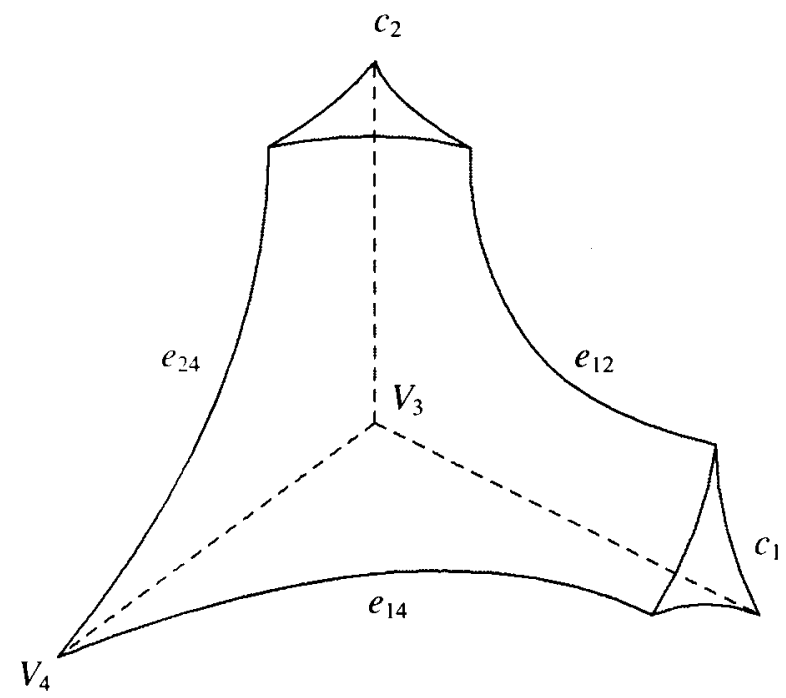

FIGURE 1. A truncated tetrahedron

We begin by outlining the classification, up to isometry, of all the Coxeter truncated tetrahedra with between one and four truncations. This is a fairly straightforward application of Andreev's theorem. The truncated tetrahedra can be grouped naturally into a finite number of infinite families.

In the next section we consider the question of finding the fewest number of generators required to generate the orientation-preserving subgroups of these groups. In most cases this is no more than two fewer than the number of faces of the associated polyhedron. This problem naturally leads to the same question for Coxeter groups in general, and in particular for extended triangle groups, and we give a number of results in this area.

In Section 4 we indicate how to calculate the volume of a truncated tetrahedron, and so the co-volume of the associated reflection group.

In Section 5 we outline how to find low index torsion-free subgroups of each group in two related families of reflection groups, $\Gamma^{0}(q)$, and $\tilde{\Gamma}^{0}(q)(7 \leq q<\infty)$. We omit the detailed proofs here as they require extensive tables, which we do not include 
(available on request from the author, or see [8]).

In the last section we use these results to construct low volume manifolds, and manifolds with totally geodesic boundary. In particular, we present a sequence of manifolds with totally geodesic boundary of any prescribed genus $g \geq 2$, which we conjecture to be of least volume among such manifolds (this is known to be true for $g=2[6])$.

This paper is essentially a chapter of my $\mathrm{Ph} . \mathrm{D}$. thesis [8]. I thank my supervisor G. J. Martin, M. D. E. Conder, B. Everitt and the referee for helpful discussions and advice.

1.1. Notation We denote the four vertices of a tetrahedron by $v_{i}(1 \leq i \leq 4)$ and the face opposite $v_{i}$ by $f_{i}$. In the case of a truncated tetrahedron we suppose that the $k(1 \leq k \leq 4)$ vertices cut off are $v_{i}$ for $1 \leq i \leq k$. The new face formed by cutting off $v_{i}$ will be denoted by $c_{i}$. The edge joining $v_{i}$ (or $c_{i}$ ) to $v_{j}$ (or $c_{j}$ ) will be denoted by $e_{i j}$ and $\alpha_{i j}=\pi / m_{i j}$ will denote the dihedral angle along this edge. This terminology is illustrated (for the case $k=2$ ) in Figure 1 .

Unless stated otherwise, angle, in this paper will always refer to a dihedral angle between two faces of a polyhedron.

\section{Classification}

All the finite volume hyperbolic Coxeter polyhedra of a given combinatorial type (other than tetrahedra) can be found using Andreev's theorem [1]. It follows from this result that there exists a tetrahedron $\mathscr{M}$ with $k$ truncations and designated angles $\alpha_{i j}$ if and only if

$$
\begin{array}{ll}
\sum_{j \neq i} \alpha_{i j}<\pi & (i \leq k) \\
\sum_{j \neq i} \alpha_{i j} \geq \pi & (i>k),
\end{array}
$$

(with equality holding in (2) exactly when the vertex $v_{i}$ is ideal) and, when $k=1$,

$$
\alpha_{23}+\alpha_{24}+\alpha_{34}<3 \pi / 2 .
$$

From this we can see that all the Coxeter truncated tetrahedra, for a given $k$, can be classified into a finite number of families, in each of which the angles along some edges are fixed, while the angles along each other edge $e$ are bounded above, and may be made arbitrarily small. Clearly $e$ has this property exactly when the angles along the four edges that meet it are all fixed at $\pi / 2$. We refer to an angle along such an edge as a parameter. For example, when $k=1$, by (3) and (1), there can 
be at most one parameter in a family and it must be one of the $\alpha_{1 j}$. By symmetry we may assume it is $\alpha_{14}$; then $m_{24}=m_{34}=2$. There are 23 families of this kind, which are listed in Table 1. This table lists the remaining values of $m_{i j}$, the smallest possible value of $q=m_{14}$, the number of cusps the prisms in this family have, and the smallest number of generators required to generate the orientation preserving subgroup of the associated reflection group (discussed in the next section). There are also 16 individual Coxeter prisms (no parameters). For $k=2$, and 3, there are 56 and 6 families, respectively, each with between two and four parameters, while for $k=4$ there is a single six-parameter family. The routine details of this classification are omitted here. Tables listing information about these families, and their associated reflection groups, are available from the author.

\begin{tabular}{|c||c|c|c|c|c|c|}
\hline$\sharp$ & $m_{12}$ & $m_{13}$ & $m_{23}$ & Min. q & Cusps & $\sharp$ Generators \\
\hline \hline 1 & 2 & 3 & 3 & 7 & 0 & 3 \\
\hline 2 & 2 & 4 & 3 & 5 & 0 & 3 \\
\hline 3 & 2 & 5 & 3 & 4 & 0 & 3 \\
\hline 4 & 3 & 3 & 3 & 4 & 0 & 3 \\
\hline 5 & 3 & 4 & 3 & 3 & 0 & 3 \\
\hline 6 & 3 & 5 & 3 & 3 & 0 & 3 \\
\hline 7 & 4 & 4 & 3 & 3 & 0 & 3 \\
\hline 8 & 4 & 5 & 3 & 2 & 0 & 3 \\
\hline 9 & 5 & 5 & 3 & 2 & 0 & 3 \\
\hline 10 & 2 & 3 & 4 & 7 & 0 & 3 \\
\hline 11 & 3 & 3 & 4 & 4 & 0 & $4(3 \mid \mathrm{q}), 3(3 \nmid \mathrm{q})$ \\
\hline 12 & 2 & 3 & 5 & 7 & 0 & 3 \\
\hline 13 & 3 & 3 & 5 & 4 & 0 & 3 \\
\hline 14 & 2 & 6 & 3 & 4 & 1 & 3 \\
\hline 15 & 3 & 6 & 3 & 3 & 1 & 3 \\
\hline 16 & 4 & 6 & 3 & 2 & 1 & 3 \\
\hline 17 & 5 & 6 & 3 & 2 & 1 & 3 \\
\hline 18 & 2 & 4 & 4 & 5 & 1 & 3 (q odd), 4(q even) \\
\hline 19 & 3 & 4 & 4 & 3 & 1 & $4(?)$ \\
\hline 20 & 2 & 3 & 6 & 7 & 1 & 3 \\
\hline 21 & 6 & 6 & 3 & 3 & 2 & 3 \\
\hline 22 & 4 & 4 & 4 & 3 & 2 & $4(\mathrm{q}$ even), 4(?)(q odd) \\
\hline 23 & 3 & 3 & 6 & 4 & 2 & $4(3 \mid \mathrm{q}), 3(3 \nmid \mathrm{q})$ \\
\hline
\end{tabular}

TABLE $1 . k=1$ one parameter: angles, generators, cusps 


\section{Presentations and generators}

For each of the truncated tetrahedra described in the previous section there is a corresponding group generated by the reflections in its faces. We denote this group by $\Gamma\left(m_{12}, m_{13}, m_{14}, m_{23}, m_{24}, m_{34}\right)$, where the $\pi / m_{i j}$ are the angles of the truncated tetrahedron in question. We will usually abbreviate this to $\Gamma(\mathbf{m})$ or simply to $\Gamma$. In this section we consider presentations for these groups and their orientation preserving subgroups, and attempt to find small sets of generators for them. Obviously, when there are $k$ truncations, $\Gamma$ can be generated by $k+4$ generators- the reflections themselves- and it is easy to show that the orientation preserving subgroup $\Gamma^{0}$ can always be generated by $k+3$ generators. In fact, as Conder and Martin [3] have found, in the case $k=1, k+2$ generators usually suffice. We show that this remains true in most of the cases $k>1$. On the way we find a number of interesting results about generators for general Coxeter groups and, in particular, extended triangle groups.

A presentation for each $\Gamma(\mathbf{m})$ is given by Poincaré's polyhedral theorem (see, for example, Maskit [10, Theorem $H$ 11]) (from which it also follows that each of these groups is discrete). This presentation takes the form $\Gamma(\mathbf{m})=(X ; R)$, where $X=\left\{f_{1}, f_{2}, f_{3}, f_{4}, c_{1}, \ldots, c_{k}\right\}$ (here we have used the same symbol to denote reflection through a face as for the face itself), and $R$ contains the following relators:

$$
\begin{gathered}
f_{i}^{2} \quad(1 \leq i \leq 4), \quad c_{i}^{2} \quad(1 \leq i \leq k) \\
\left(f_{i} f_{j}\right)^{m_{p 4}} \quad(1 \leq i<j \leq 4) \\
\left(f_{i} c_{j}\right)^{2} \quad(1 \leq i \leq 4) \quad(1 \leq j \leq k, i \neq j)
\end{gathered}
$$

where $\{p, q\}=\{1,2,3,4\} \backslash\{i, j\}$.

In particular, the reflection groups $\Gamma(\mathbf{m})$ are all Coxeter groups. Given a presentation $(X ; R)$ for any Coxeter group, we term the elements of $X$ canonical generators and the product of any two of these, if of finite order, edge relators. In the case of reflection groups, the canonical generators are thus reflections and the edge relators, rotations about edges. In the sequel we will assume $X$ is finite.

Suppose that the canonical generators for $C$ with presentation $(X ; R)$ are $a_{1}, a_{2}, \ldots$, $a_{n}$. Let $x_{i}=a_{i} a_{i+1}(1 \leq i \leq n-1)$. We let $C^{0}$ denote the (index 2) subgroup of $C$ generated by $X^{\prime}$. A presentation for $C^{0}$ is given by $\left(X^{\prime} ; R^{\prime}\right)$, where $X^{\prime}=\left\{x_{i}: 1 \leq\right.$ $i \leq n-1\}$ and $R^{\prime}$ is obtained from $R$ by omitting the relators of the form $a_{i}{ }^{2}=1$ and rewriting the rest in terms of the $x_{i}$.

The group $\Gamma^{0}(\mathbf{m})$ is always generated by $k+3$ elements. The following lemma, proved by Conder and Martin [3], shows that $k+2$ generators usually suffice.

LEMMA 1. If $\alpha, \beta, \gamma, \delta$ are group elements satisfying the relations

$$
\alpha^{2}=\beta^{2}=\gamma^{2}=\delta^{2}=(\alpha \beta)^{2}=(\beta \gamma)^{2}=(\gamma \delta)^{2}=(\delta \alpha)^{n}=1
$$


where $n$ is odd, then $\langle\alpha \beta, \beta \gamma, \gamma \delta\rangle$ is a 2-generator group, generated for example by $\gamma \alpha$ and $\beta \delta$.

Suppose a polyhedron with $p$ faces has among them four, say $a_{1}, a_{2}, a_{3}, a_{4}$, such that, for each $i \leq 3, a_{i}$ and $a_{i+1}$ meet at an angle of $\pi / 2$, while $a_{4}$ and $a_{1}$ meet at angle $\pi / n$ ( $n$ odd). In this case the above lemma shows that $\left\langle a_{1} a_{2}, a_{2} a_{3}, a_{3} a_{4}\right\rangle$ is a 2-generator group, and hence that the orientation preserving group for this polyhedron is $(p-2)$-generator.

In the case of the truncated tetrahedra, there are four such faces if either of the following conditions hold:

(1) For some $i \leq k$ there are 2 faces $f_{j}, f_{i}$, adjoining $f_{i}$ at angles $\pi / 2$ and $\pi / n(n$ odd), respectively; in this case, let $\left(a_{1}, a_{2}, a_{3}, a_{4}\right)=\left(f_{i}, f_{j}, c_{i}, f_{l}\right)$, that is, there exist $i \leq k$ and $j, l, m$ distinct from $i$ and each other such that $m_{j l}=2$ and $m_{l m}$ is odd.

(2) For some $i \leq k$ one of the three angles between the faces adjoining $c_{i}$ is $\pi / 2$ and another is $\pi / n$ ( $n$ odd); in this case $\left\{a_{1}, a_{2}, a_{3}, a_{4}\right\}$ comprises $c_{i}$ and its three adjoining faces, that is, there exist $i \leq k$ and $j, l$ such that $m_{i j}=2$ and $m_{i l}$ is odd.

For most truncated tetrahedra, at least one of these conditions is satisfied. For example, among those listed in Table 1, the only exceptions are those numbered 11 , $19,22,23$ and, when the parameter $m_{14}$ is even, 18.

To deal with the exceptional cases further we prove some more results on triangle groups. In what follows $\Delta=\Delta(p, q, r)$ will denote the extended triangle group, with presentation,

$$
\left\langle a, b, c ; a^{2}=b^{2}=c^{2}=(a b)^{p}=(b c)^{q}=(a c)^{r}=1\right\rangle .
$$

The content of the next theorem is implicit in [3, Lemma 2.1 and argument preceding Theorem 2.3].

THEOREM 2. The group $\Delta$ is a 2-generator group if one of $p, q, r$ is 2 and another is odd, or if two of $p, q, r$ are 3 and the third is not a multiple of 3 .

Proof. Suppose that the first condition holds. We may assume that $p=2$, and $q$ is odd. The group $\langle a c, b\rangle$ contains $[a c, b]=a c b c a b=a c b c b a$. Taking the $(q+1) / 2$ th power of this element gives $a c b a$, whence $b a$ and so also $a$ and $c$ are in $\langle a c, b\rangle$, which is thus the entire group.

In the second case, we may assume that $p=q=3$ and $r \not \equiv 0 \bmod 3$. The group $\langle a b c, b\rangle$ then contains $(a b c) b(c b a)=a c a$ and $(c b a) b(a b c)=c a c$, whence it also contains acacac, a power of which is $a c$. It then follows that $\langle a b c, b\rangle$ also contains $(a b c)(c a)=a b a, b a b a=a b$ and hence $a$ and $c$, so that again $\langle a b c, b\rangle=\Delta$.

We conjecture that the converse to the above theorem also holds: That is, 
CONJECTURE 3. The group $\Delta$ is a 2-generator group only if one of $p, q, r$ is 2 and another is odd, or two of $p, q, r$ are 3 and the third is not a multiple of 3 .

THEOREM 4. If $P$ represents the $k$-fold free product of $Z_{2}$ with itself, and $C$ is a Coxeter group with $n$ canonical generators, and for which the powers of the edge relators have a common factor greater than one, then the group $(P \oplus C)^{0}$ requires at least $n+k-1$ generators.

PrOOF. Let $a_{1}, a_{2}, \ldots, a_{k}$ be the canonical generators of $P$, and $b_{1}, b_{2}, \ldots, b_{n}$ be those of $C$. Then the products $a_{i} a_{i+1}(1 \leq i<k), b_{i} b_{i+1}(1 \leq i<n)$ and $a_{1} b_{1}$ generate $(P \oplus C)^{0}$. By assumption the powers of the edge relators of $C$ have a common factor $h>1$. Let $G$ be the direct sum of $n+k-2$ copies of the cyclic group $Z_{h}$, and let $G^{\prime}$ be the $Z_{2}$ extension of $G$ obtained by appending to $G$ an element $t$ of order 2 which inverts every element of $G$. We determine a homomorphism of $(P \oplus C)^{0}$ onto $G^{\prime}$, by mapping the $n+k-2$ generators $a_{i} a_{i+1}$ and $b_{i} b_{i+1}$ to the generators of the factors of $G$, and $a_{1} b_{1}$ to $t$. This is readily verified to give a well-defined homomorphism. Since $G^{\prime}$ requires at least $n+k-1$ generators, the same is true of $(P \oplus C)^{0}$.

REMARK. Since easily, $\left(Z_{2} \oplus C\right)^{0}$ is isomorphic to $C$, it follows, when $C$ satisfies the hypotheses of this corollary, that $C$ requires $n$ generators.

We now apply these results to the groups $\Gamma^{0}(\mathbf{m})$.

COROLLARY 5. If $k=1$ and $m_{23}, m_{24}$ and $m_{34}$ are even and $m_{12}, m_{13}, m_{14}$ have a common factor greater than one then $\Gamma^{0}(\mathbf{m})$ requires four generators.

PROOF. Since $m_{23}, m_{24}$ and $m_{34}$ are even, $\left(Z_{2} * Z_{2}\right) \times \Delta\left(m_{12}, m_{13}, m_{14}\right)$ is a quotient group of $\Gamma$, and the corollary follows immediately from the above result.

On the other hand,

LEMMA 6. If, for some $i \leq k, \Delta\left(m_{i j}, m_{i k}, m_{i l}\right)$ is two-generator, where $\{j, k, l\}=$ $\{1,2,3,4\} \backslash\{i\}$, then $\Gamma^{0}(\mathbf{m})$ is $(k+2)$-generator.

PROOF. For convenience we assume $i=1$, then $\Gamma^{0}$ is generated by $x=c_{1} f_{2}$, $y=c_{1} f_{3}, z=c_{1} f_{4}, w=c_{1} f_{1}$ and $v_{i}=c_{1} c_{i}(1<i \leq k)$. Rewriting the relators from the presentation for $\Gamma$ in terms of the new generators, it is clear that the subgroup $\langle x, y, z\rangle$ is the extended triangle group $\Delta\left(m_{i j}, m_{i k}, m_{i l}\right)$, which is two-generator by hypothesis, whence $\Gamma^{0}$ is $(k+2)$-generator. 
Using Theorem 2 and the above two results we can determine whether or not three generators suffice, for most of the outstanding groups $\Gamma^{0}$ given in Table 1. Only families 19, with $q=m_{14}$ odd, and 22 remain unresolved but, by Corollary 5, the groups from these families will require four generators if the extended triangle groups $\Delta(3,4, q)$ and $\Delta(4,4, q)$ respectively, require three generators, which will be true if Conjecture 3 holds. The above results can also be used to show that $\Gamma^{0}$ requires only $k+2$ generators in most cases when $k>1$. The most obvious exceptions are the groups for which each of the $m_{i j}$ is even, when replacing each of the $m_{i j}$ by 2 and abelianizing gives the $(k+4)$-fold product of $Z_{2}$ as a quotient of $\Gamma$. More details may be found in [8].

\section{Volumes}

We now show how to calculate the volume of a truncated tetrahedron, and hence the co-volumes of the groups $\Gamma(\mathbf{m})$ and $\Gamma^{0}(\mathbf{m})$.

By Schläfli's equation (see, for example, [14, Chapter 7, 2.2]), if $P$ is a hyperbolic polyhedron of given combinatorial type with dihedral angles $\theta_{1}, \theta_{2}, \ldots, \theta_{m}$, then its volume, $V=V\left(\theta_{1}, \theta_{2}, \ldots, \theta_{m}\right)$ has derivatives:

$$
\partial V / \partial \theta_{i}=-l_{i} / 2 \quad(1 \leq i \leq m)
$$

where $l_{i}$ is the length of the edge along which the angle $\theta_{i}$ occurs.

Given this result, we may find $V$ as a function of $\boldsymbol{\theta}=\left(\theta_{1}, \theta_{2}, \ldots, \theta_{m}\right)$ provided we know the edge lengths as a function of $\boldsymbol{\theta}$ along with the volume at some particular initial value $\theta_{0}$ of $\theta$. The volume at $\theta=\theta_{1}$ is then given by the line integral:

$$
V\left(\theta_{1}\right)=V\left(\theta_{0}\right)+\int_{\theta_{0}}^{\theta_{1}} \nabla V \cdot d \theta .
$$

We now apply these results to truncated tetrahedra. Throughout, $\theta_{i j}$ will be the variable of integration corresponding to the angle $\alpha_{i j}$, and $\alpha$ will denote the vector $\left(\alpha_{12}, \ldots, \alpha_{34}\right)$.

When $k=1$ we vary only the three angles $\theta_{23}, \theta_{24}$ and $\theta_{34}$. We $\operatorname{set} \boldsymbol{\theta}=\left(\theta_{23}, \theta_{24}, \theta_{34}\right)$. The $\theta_{1 j}(2 \leq j \leq 4)$ will be fixed at value $\alpha_{1 j}$ while the remaining three angles are all $\pi / 2$. For the initial value we set:

$$
\theta=\boldsymbol{\alpha}_{0}=(\pi / 2, \pi / 2, \pi / 2) .
$$

This choice of angles determines a degenerate prism of zero volume. Faces $f_{1}$ and $c_{1}$ coincide, both being triangles with angles $\theta_{12}, \theta_{13}$ and $\theta_{14}$. The volume of the prism is 
now given by:

$$
V=V\left(\alpha_{12}, \alpha_{13}, \alpha_{14}, \alpha_{23}, \alpha_{24}, \alpha_{34}\right)=\int_{\alpha_{0}}^{\alpha_{1}} \nabla V \cdot d \theta
$$

where $\boldsymbol{\alpha}_{1}=\left(\alpha_{23}, \alpha_{24}, \alpha_{34}\right)$.

The length $l_{i j}$ of the edge $e_{i j}$ is given by:

$$
l_{i j}=\operatorname{arcsinh} \sqrt{-|G|\left|G_{i j}^{i j}\right| /\left|G_{i}^{i}\right|\left|G_{j}^{i}\right|}
$$

(see, for example, equation (16) of [9]) where $G$ is the Gram matrix corresponding to faces $f_{1}, f_{2}, f_{3}, f_{4}$. In view of (4) and (8), evaluating the line integral in (7) is a straightforward numerical calculation. For simplicity, the path of integration may be taken as a line segment joining the endpoints.

In the cases where the angle $\alpha_{14}$ is a parameter, the angles $\alpha_{24}$ and $\alpha_{34}$ are both $\pi / 2$. Hence, in this case, we may calculate the volume by varying $\alpha_{23}$ only. This method also works in the degenerate case $\alpha_{14}=0$.

The volume of the prism is the co-volume of the corresponding reflection group $\Gamma(\mathbf{m})$ and these volumes are doubled to get the co-volumes of the index two subgroup $\Gamma^{0}(\mathbf{m})$.

When $2 \leq k \leq 3$ we can calculate volumes using induction on $k$. In these cases we vary only the angle $\phi=\theta_{12}$, the other angles remaining fixed.

Let $\phi_{1}=\alpha_{12}$ and $\phi_{0}$ be the least value of $\phi$ for which at least one of $\phi+\alpha_{13}+\alpha_{14}$, $\phi+\alpha_{23}+\alpha_{24}$ is $\pi$. Geometrically, $\phi_{0}$ is obtained by continuously increasing $\phi$ until one of the truncations at $v_{1}$ and $v_{2}$ becomes instead an ideal vertex (which may be viewed as a truncation 'at infinity'). If we let $\boldsymbol{\alpha}^{\prime}$ be the vector obtained by replacing $\alpha_{12}$ in $\alpha$ by $\phi_{0}$ then (4) and (7) give

$$
\begin{aligned}
V(\boldsymbol{\alpha}) & =V\left(\boldsymbol{\alpha}^{\prime}\right)+\int_{\phi_{1}}^{\phi_{1}} \frac{\partial V}{\partial \phi} d \phi \\
& =V\left(\boldsymbol{\alpha}^{\prime}\right)+\frac{1}{2} \int_{\phi_{1}}^{\phi_{1}} l_{12} d \phi .
\end{aligned}
$$

In the degenerate case, $\phi_{1}=\alpha_{12}=0$, the integral (10) is improper.

Since $\boldsymbol{\alpha}^{\prime}$ determines a tetrahedron with $(k-1)$ or fewer truncations, its volume can be calculated by the induction hypothesis.

To complete the calculation we need only find $l_{12}$ as a function of $\phi$. Since $2 \leq k \leq 3, f_{3}$ is a pentagon with sides $a_{1}, l_{12}, a_{2}, l_{24}$ and $l_{14}$, where $a_{i}(i=1,2)$ is the edge between $f_{3}$ and $c_{i}$. If we let $\psi$ be the face angle between edges $l_{14}$ and $l_{24}$, a formula of Beardon [2, Theorem 7.18.1(i)] gives

$$
\cosh \left(l_{12}\right)=\frac{\cosh \left(a_{1}\right) \cosh \left(a_{2}\right)+\cos (\psi)}{\sinh \left(a_{1}\right) \sinh \left(a_{2}\right)} .
$$


The spherical cosine rule applied to a small sphere centred at $v_{4}$ gives

$$
\cos (\psi)=\frac{\cos \left(\theta_{14}\right) \cos \left(\theta_{24}\right)+\cos \left(\theta_{34}\right)}{\sin \left(\theta_{14}\right) \sin \left(\theta_{24}\right)}
$$

while the hyperbolic cosine rule applied to $c_{1}$ (whose face angles are just the dihedral angles $\left.\phi, \theta_{13}, \theta_{14}\right)$ gives

$$
\cosh \left(a_{1}\right)=\frac{\cos (\phi) \cos \left(\theta_{14}\right)+\cos \left(\theta_{13}\right)}{\sin (\phi) \sin \left(\theta_{14}\right)}
$$

and symmetrically

$$
\cosh \left(a_{2}\right)=\frac{\cos (\phi) \cos \left(\theta_{24}\right)+\cos \left(\theta_{23}\right)}{\sin (\phi) \sin \left(\theta_{24}\right)}
$$

whence $\sinh \left(a_{1}\right), \sinh \left(a_{2}\right)$ can also be calculated. Using (11)-(14), we may now calculate numerically $l_{12}$ as a function of $\phi$ and so the integral (10).

When $k=4$, a similar approach could be used to reduce the calculation to the case $k=3$. However it is more convenient to proceed as follows. In (7) set $\boldsymbol{\alpha}_{0}=(\pi / 3, \pi / 3, \pi / 3, \pi / 3, \pi / 3, \pi / 3)$. Thus the initial polyhedron is ideal and so its volume is easily calculated by the well-known formula

$$
\operatorname{volume}(\Delta)=3 \eta(\pi / 3)
$$

where $\eta(\theta)$ is the Lobachevsky function defined by

$$
\eta(\theta)=-\int_{0}^{\theta} \log |2 \sin (u)| d u
$$

(see, for example, [11, p. 18 Lemma 2]). Combining (7) and (15) gives

$$
V=V(\boldsymbol{\alpha})=3 \eta(\pi / 3)+\int_{\boldsymbol{\alpha}_{0}}^{\boldsymbol{\alpha}} \nabla V \cdot \boldsymbol{d} \boldsymbol{\theta} .
$$

Again we may take the integration path to be a line segment. It is easy to verify that $\theta$ at any interior point of this path, determines a tetrahedron with four truncations. Hence each $f_{i}$ is a hexagon (if $k<4$ this is not generally true, which is why this approach does not work in these cases). To calculate the integral in (17) it is again simply a question of calculating the lengths $l_{i j}$ as a function of $\theta$.

As an example, we show how to calculate $l_{12}$, the other edge lengths all being found similarly. The required edge length $l_{12}$ is the length of one of the sides of $f_{4}$, the others being in turn, $b_{2}, l_{23}, b_{3}, l_{13}$ and $b_{1}$, where $b_{i}(1 \leq i \leq 3)$ is the length of the edge between $f_{4}$ and $c_{i}$. All the angles in this hexagon are right angles which allows us to apply another formula of Beardon's [2, Theorem 7.19.2] which gives

$$
\cosh \left(l_{12}\right)=\frac{\cosh \left(b_{1}\right) \cosh \left(b_{2}\right)+\cosh \left(b_{3}\right)}{\sinh \left(b_{1}\right) \sinh \left(b_{2}\right)}
$$

and, as in (13), (14), the $\cosh \left(b_{i}\right)$, and hence also the $\sinh \left(b_{i}\right)$, can be expressed in terms of $\theta$ by using the cosine rule on the triangular face $c_{i}$. 


\section{Torsion-free subgroups}

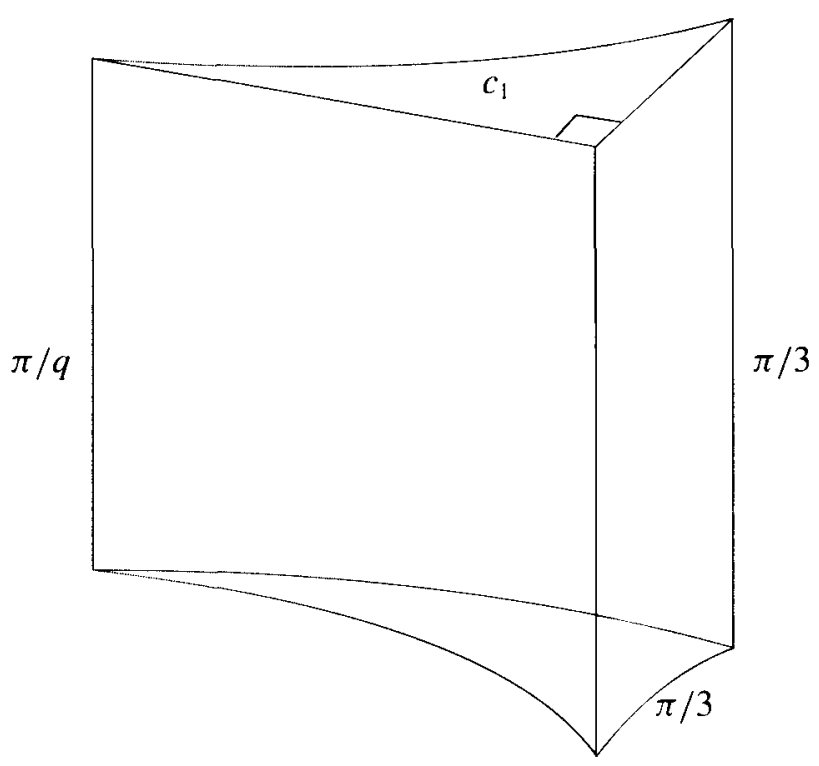

FIGURE 2. The prism $P(q)$

In this section we find low index torsion-free subgroups of two related families of reflection groups, $\Gamma^{0}(q)$ and $\tilde{\Gamma}^{0}(q)$, where, $\Gamma^{0}(q)=\Gamma^{0}(2,3, q, 3,2,2)$ has presentation

$$
\left\langle a, p, c, e \mid a^{2}=p^{2}=c^{3}=e^{2}=(a c)^{3}=(p c)^{2}=(a p)^{q}=(a c e)^{2}=(p c e)^{2}\right\rangle
$$

and $\tilde{\Gamma}^{0}(q)$ is the subgroup $\langle a, p, c\rangle$ of $\Gamma^{0}(q)$. Geometrically, $\Gamma^{0}(q)$ is the orientationpreserving subgroup of the reflection group of the prism $P(q)$ pictured in Figure 2. (The dihedral angles of this prism are all $\pi / 2$, except where indicated otherwise.)

The subgroup $\tilde{\Gamma}^{0}(q)$, is thus the orientation-preserving subgroup of the reflection group of the 'open' prism obtained by omitting the truncating face $c_{1}$ from $P(q)$.

The lowest common multiple of the orders of the finite subgroups of both $\Gamma^{0}(q)$ and $\tilde{\Gamma}^{0}(q)$ is $\operatorname{LCM}(12,2 q)$. It is well known that this number must divide the index of any torsion-free subgroup of these groups.

DEFINITION. If $G$ is a Coxeter group and $G^{0}$ acts on a set $X$, then this group action is said to be semiregular, and $X$ a semiregular $G^{0}$-set, if the permutation effected by the action of each edge relator is semiregular (having all cycles of equal length) and this cycle length is equal to the order of the relator. 
It is well known that every finite-order element of a hyperbolic reflection group (in two or three dimensions) is conjugate to a power of an edge relator (see, for example, [3]). We can thus find torsion-free subgroups of $\tilde{\Gamma}^{0}(q)$ using the following well-known result (see, for example, [13]).

THEOREM 7. Let $C$ be a Coxeter group with the property that any $g \in C^{0}$ is of finite order if and only if it is conjugate to a power of an edge relator. The group $C^{0}$ has a torsion-free subgroup of index $n$ if and only if there is a transitive semiregular action of $C^{0}$ on a set $S$ of $n$ elements.

Our basic approach has been to find semiregular $\tilde{\Gamma}^{0}(q)$-sets for small values $q_{1}, q_{2}, \ldots, q_{n}$ of $q$ and then 'splice' these sets together in such a way as to construct a transitive semiregular $\tilde{\Gamma}^{0}\left(q_{1}+q_{2}+\cdots+q_{n}\right)$-set, and apply the above theorem. In this way we can find torsion-free subgroups of $\tilde{\Gamma}^{0}(q)$ of the smallest possible index for every $q \geq 6$ (Theorem 9). Adapting the arguments also gives torsion-free subgroups of $\Gamma^{0}(q)$ of the smallest possible index in most cases when $q$ is a multiple of 4 (Theorem 12). As stated in the introduction, we omit details of the construction.

We introduce a set of Coxeter groups which slightly generalize the $\tilde{\Gamma}^{0}(q)$.

DEFINITION. Define $G(q)$ by

$$
G(q)=\left\{a, p, c \mid a^{2}=p^{2}=c^{m}=(p c)^{2}=(a c)^{n}=(a p)^{q}\right\} .
$$

We have $G(q)=\tilde{\Gamma}^{0}(q)$ when $m=n=3$. By a slight abuse of notation, we will use the same letters $a, p$ and $c$ to refer to the generators of $G(q)$ for different values of $q$.

The following results are concerned with actions of $G(q)$ on finite sets. In general, if a group $G$ acts on a set $X$ we refer to a set of the form $\left\{g^{n} x \mid n \in Z\right\}(x \in X, g \in G)$ as a $g$-cycle in $X$. If $g$ is of finite order $m$ then the sequence $g^{m_{1}} x, g^{m_{2}} x, \ldots, g^{m_{k}} x$, with $0 \leq m_{i}<m$ for each $i$, is said to be in cyclic order if, for some $j \leq k$,

$$
0 \leq m_{j}<m_{j+1}<\cdots m_{k}<m_{1}<m_{2}<\cdots<m_{j-1}<m .
$$

Clearly a sequence is in cyclic order if and only if any cyclic permutation of it is also.

DEFINITION. Let $X_{1}$ be a $G\left(q_{1}\right)$-set and $X_{2}$ a $G\left(q_{2}\right)$-set, for which the group action is semiregular. Let $\Sigma_{1} \subseteq X_{1}, \Sigma_{2} \subseteq X_{2}$ both be unions of $c$-cycles which are disjoint from their images under $p$. Define $C_{1}=\Sigma_{1} \cup p\left(\Sigma_{1}\right), C_{2}=\Sigma_{2} \cup p\left(\Sigma_{2}\right)$. We define an equivalence relation as follows. The pairs $\left(X_{1}, \Sigma_{1}\right)$ and $\left(X_{2}, \Sigma_{2}\right)$ are equivalent if there is a bijection $\psi: C_{1} \rightarrow C_{2}$ for which

(1) $\psi\left(\Sigma_{1}\right)=\Sigma_{2}$.

(2) $\psi(c x)=c \psi(x) \quad \psi(p x)=p \psi(x)$ 
(3) Two points in $C_{1}$ are in the same ap-cycle if and only if their images under $\psi$ are.

(4) If $H_{1}$ is an ap cycle in $X_{1}$ and $x_{1}, x_{2}, \ldots, x_{m}$ are the points of $H_{1} \cap C_{1}$ in cyclic order, then the points $\psi\left(x_{1}\right), \psi\left(x_{2}\right), \ldots, \psi\left(x_{m}\right)$ are also in cyclic order.

LEMMA 8 (Splicing). For $1 \leq i \leq n$, let $q_{i}$ be a positive integer, $X_{i}$ a semiregular $G\left(q_{i}\right)$-set, and $\Sigma_{i} \subseteq X_{i}$ a union of c-cycles for which $\Sigma_{i}$ and $p\left(\Sigma_{i}\right)$ are mutually disjoint. Let $C_{i}=\Sigma_{i} \cup p\left(\Sigma_{i}\right)$.

Suppose also that the $\left(X_{i}, \Sigma_{i}\right)$ are all mutually equivalent, and that, for each apcycle $H \subseteq X_{1}$, the difference d between the cardinalities $m_{1}$ of $H \cap \Sigma_{1}$ and $m_{2}$ of $H \cap p\left(\Sigma_{1}\right)$ is relatively prime to $n$. The disjoint union of the $X_{i}$ can then be made into a semiregular $G(q)$-set, where $q=\sum q_{i}$.

If $X_{i}$ is a transitive $G\left(q_{i}\right)$-set for some $i$, then $X$ is a transitive $G(q)$-set.

SKETCH OF PROOF. We may assume that the sets $X_{i}$ are pairwise disjoint. For $1 \leq i \leq n-1$ let $\left(X_{i}, \Sigma_{i}\right)$ and $\left(X_{i+1}, \Sigma_{i+1}\right)$ be equivalent via the map $\psi_{i}$, and define $\psi_{n}=\left(\psi_{n-1} \circ \psi_{n-2} \circ \cdots \circ \psi_{1}\right)^{-1}$. Let $X=\bigcup_{i=1}^{n} X_{i}$.

For $x \in X_{i}$ we denote the image of $x$ under the given action of $g \in G\left(q_{i}\right)$ by $g *_{i} x$. We claim that the maps $a, p, c: X \rightarrow X$ given by

$$
\begin{array}{ll}
a x=a *_{i} x, & x \in X_{i} \\
c x=c *_{i} x, & x \in X_{i}
\end{array}
$$

and

$$
p x= \begin{cases}p *_{i+1} \psi_{i}(x), & x \in \Sigma_{i} \\ p *_{i-1} \psi_{i-1}^{-1}(x), & x \in p\left(\Sigma_{i}\right) \\ p *_{i} x, & x \in X_{i} \backslash C_{i}\end{cases}
$$

determine a semiregular action of $G(q)$ on $X$.

The action of $p$ on $X$ is shown schematically in Figure 3, where dotted lines represent the original action of $p$ on the individual $X_{i}$, and solid lines represent the action of $p$ on $X$. The $n$ sets are joined this way in a loop. One can then show that the permutations on $X$ given by $a, c, p, a c, c p$ and $a p$ have the required cycle structure (all but the last being trivial). The last statement of the theorem is also readily proved.

THEOREM 9. For each $q \geq 7$, the group $\tilde{\Gamma}^{0}(q)$ has a torsion-free subgroup of index $\operatorname{LCM}(12,2 q)$, and $\tilde{\Gamma}^{0}(6)$ has a torsion-free subgroup of index 24 . These indices are the smallest possible in each case. 


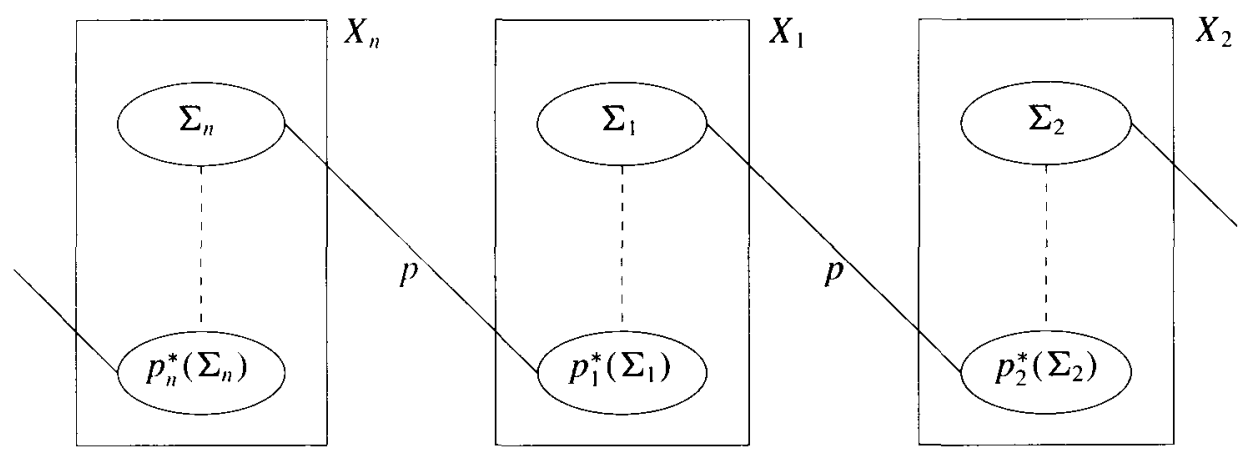

FIGURE 3. Action of $p$ on $X$

The above is proved using Lemma 8 , and some directly computed group actions. As an illustrative example we prove the case $q \equiv 0 \bmod 6, q \geq 12$.

Given any transitive semiregular $\tilde{\Gamma}^{0}(q)$ actions on a set of $2 q$ elements, we may normalize by assuming that the set is $[2 q]=\{1,2, \ldots, 2 q\}$, and that the actions of $p$ and $a$ are given by,

$$
\begin{aligned}
& p x= \begin{cases}x+1 & \text { if } x \text { is odd } \\
x-1 & \text { if } x \text { is even, }\end{cases} \\
& a x= \begin{cases}x-1 & \text { if } x \text { is odd } \\
x+1 & \text { if } x \text { is even }\end{cases}
\end{aligned}
$$

\begin{tabular}{|c||c|c|c|c|c|c|c|c|c|c|c|c||}
\hline$x$ & 1 & 2 & 3 & 4 & 5 & 6 & 7 & 8 & 9 & 10 & 11 & 12 \\
\hline$c x$ & 23 & 17 & 19 & 10 & 14 & 11 & 15 & 22 & 3 & 20 & 13 & 5 \\
\hline \hline$x$ & 13 & 14 & 15 & 16 & 17 & 18 & 19 & 20 & 21 & 22 & 23 & 24 \\
\hline$c x$ & 6 & 12 & 21 & 8 & 24 & 1 & 9 & 4 & 7 & 16 & 18 & 2 \\
\hline
\end{tabular}

TABLE 2. Values of $c x: q=12$

It follows that the two ap-cycles are simply the sets of odd numbers and even numbers. It remains only to specify the action of $c$. For $q=12$ and $q=18$ respectively these are given in Tables 2 and 3.

If we let $\Sigma_{1}=\{1,23,18\}$ and $\Sigma_{2}=\{5,33,28\}$, and $\psi$ be determined by $\psi_{1}(1)=5$, $\psi_{1}(23)=33, \psi_{1}(18)=28$, then $\left([24], \Sigma_{1}\right)$ and ([36], $\left.\Sigma_{2}\right)$ are equivalent, and we 


\begin{tabular}{|c||c|c|c|c|c|c|c|c|c|c|c|c||}
\hline$x$ & 1 & 2 & 3 & 4 & 5 & 6 & 7 & 8 & 9 & 10 & 11 & 12 \\
\hline$c x$ & 35 & 7 & 9 & 25 & 33 & 27 & 36 & 1 & 26 & 4 & 19 & 31 \\
\hline \hline$x$ & 13 & 14 & 15 & 16 & 17 & 18 & 19 & 20 & 21 & 22 & 23 & 24 \\
\hline$c x$ & 15 & 16 & 22 & 21 & 30 & 24 & 32 & 12 & 14 & 13 & 17 & 29 \\
\hline \hline$x$ & 25 & 26 & 27 & 28 & 29 & 30 & 31 & 32 & 33 & 34 & 35 & 36 \\
\hline$c x$ & 10 & 3 & 34 & 5 & 18 & 23 & 20 & 11 & 28 & 6 & 8 & 2 \\
\hline
\end{tabular}

TABLE 3. Values of $c x: q=18$

may use Theorem 8 to join together $n_{1}$ copies of [24] and $n_{2}$ copies of [36] to form a semiregular $\tilde{\Gamma}^{0}(q)$-set on which $\tilde{\Gamma}^{0}(q)$ acts transitively.

Since every multiple of 6 greater than 6 itself can be expressed as $12 n_{1}+18 n_{2}$ $\left(n_{1}, n_{2} \geq 0\right)$ the proof of this case is completed.

From the above construction, we also easily obtain the following result, which will be useful in the next section.

COROLlaRY 10. In the case $q \equiv 0 \bmod 6$ of the above theorem, the subgroup $\langle a p, p c\rangle$ acts transitively on the $\tilde{\Gamma}^{0}(q)$-set $X$ constructed in the theorem.

We now consider the groups $\Gamma^{0}(q)$. To obtain a torsion-free subgroup of index $n$ of $\Gamma^{0}(q)$, it suffices to find a semiregular $\tilde{\Gamma}^{0}(q)$-set $X$ of $n$ elements and then define an action of $e$ on this set in such a way that ace, pce and $e$ are each semiregular and of order two. In practice it is easier to replace the generator $e$ by its conjugate $\pi=(c a)^{-1} e(c a)$. We have

$$
\pi c=(a c a)(a c e)(a c a)^{-1} \text { and } \pi a p a=(a c p)(p c e)(a c p)^{-1}
$$

so that the permutations effected by $\pi, \pi c$, and $\pi a p a$ are all semiregular of order two. Conversely, given $\pi$ with these properties we may define $e=(c a) \pi(c a)^{-1}$. The generator $\pi$ is slightly more convenient to use than $e$ and, as we will show in the next section, arises naturally in a geometric context.

The following is an elaboration of Lemma 8 .

COROLlarY 11. Suppose the $X_{i}$ in Lemma 8 are all $\tilde{\Gamma}^{(1}\left(q_{i}\right)$-sets that can be made into semiregular $\Gamma^{0}\left(q_{i}\right)$-sets by specifying an action of $\pi$. If furthermore for each $i, \Sigma_{i}$ and $p\left(\Sigma_{i}\right)$ are both invariant under the action of $a \pi a$, and the action of $a \pi a$ commmutes with each equivalence map $\psi_{i}: C_{i} \rightarrow C_{i+1}$, then $X$ can be made into a semiregular $\Gamma^{0}(q)$-set.

This result can be used to prove ([8]) 
THEOREM 12. If $q$ is a multiple of 4 other than $4,16,20,36$ or 48 then the group $\Gamma^{0}(q)$ has a torsion-free subgroup of index $\operatorname{LCM}(12,2 q)$.

\section{Construction of manifolds}

We now describe a direct construction of manifolds by gluing together copies of the prism $P(q)$. We begin by gluing 24 prisms together into a regular truncated tetrahedron, and then glue two or more of these tetrahedra together to construct a manifold with boundary. On the way, we obtain a nice geometric interpretation of semiregular group actions of $\tilde{\Gamma}^{0}(q)$.

Finally in some cases we can glue the boundary of one of these manifolds to itself to obtain a manifold without boundary.

The initial step - from prisms to tetrahedra - is indicated in Figure 4. Six copies of $P(q)$ are joined as shown, to give a prism-like figure with a flat top and a ridged base. The three ridges meet at vertex $v$. The link at this vertex is a spherical equilateral triangle with three angles of $2 \pi / 3$, so that four of the polyhedra pictured in Figure 4 can be fitted together to make a regular truncated tetrahedron with common dihedral angle $2 \pi / q$.

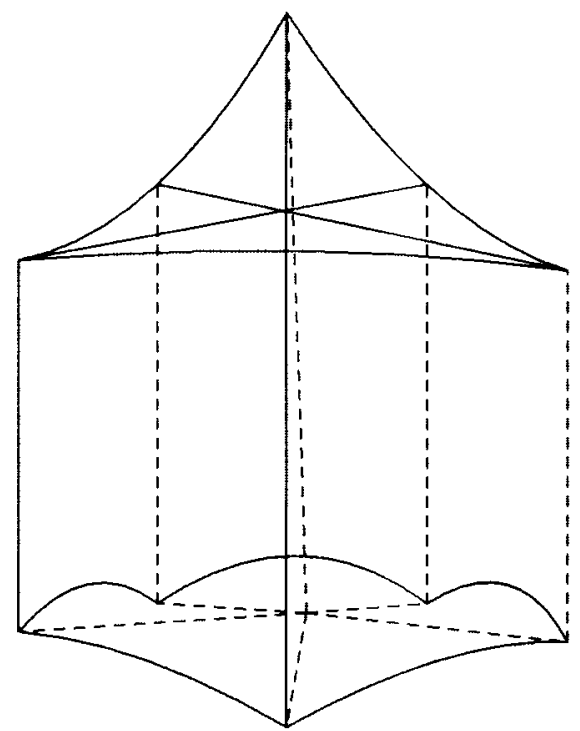

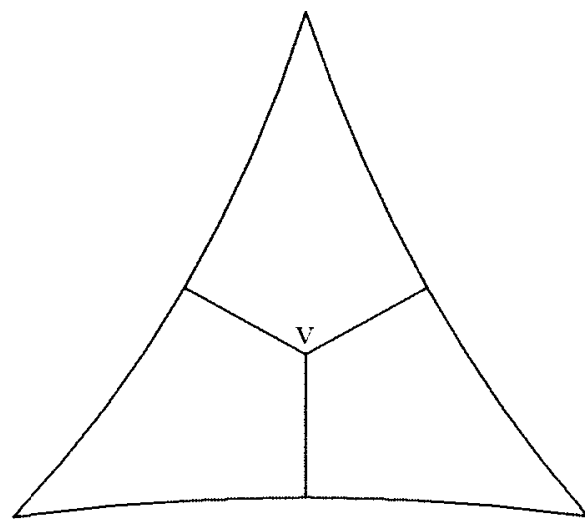

View from underneath

FIGURE 4. Gluing of $P(q)$

We construct manifolds with boundary by gluing two or more of these truncated tetrahedra together along the faces $f_{i}$, the faces $c_{i}$ (those formed by the truncations) 
then forming the boundary of the manifold. This construction is done quite explicitly by Fujii [5] for two tetrahedra in the case $q=12$. We can describe the gluing operation algebraically as follows:

Suppose $k$ truncated tetrahedra are glued together. Each tetrahedron has 6 edges where two of the faces $f_{i}$ meet. We define $E$ to be the set of edge-face pairs $(e, f)$, where $e$ is such an edge and $f=f_{i}$ one of the faces it bounds. This set has $12 k$ elements. We define the adjacency map $a$ on $E$ as the map which takes $(e, f)$ to $\left(e, f^{\prime}\right)$, where $f$ and $f^{\prime}$ are the two faces with $e$ as a boundary edge, and the cycle map $c$ by $c((e, f))=(d, f)$, where $d$ is the next edge bounding $f$ anticlockwise from $e$ (see Figure 5). Clearly $a$ and $c$ are semiregular permutations on $E$, with cycle lengths 2 and 3 respectively. It is also easy to show that $a c$ is semiregular with cycle length 3 .

Now suppose that the faces $f_{i}$ of the tetrahedra are glued together in pairs in such a way as to construct a manifold with boundary. This pairing of faces induces a third permutation $p$ on $E$. We define $p((e, f))=\left(e_{2}, f_{2}\right)$ where the gluing map takes $f$ to $f_{2}$ and $e$ to $e_{2}$ (Figure 5). Clearly $p$ is semiregular with cycle length 2 . This is also true of $p c$ (because the gluing map is orientation reversing). Finally, because each edge must be identified with $q-1$ others (so that the angle sum is $2 \pi$ ), we require $a p$ to be semiregular with cycle length $q$. We have thus obtained a semiregular action of $\tilde{\Gamma}^{0}(q)$ on $E$. Conversely any such action on a set of $12 k$ elements can be interpreted geometrically as a gluing of $k$ truncated tetrahedra to give a manifold with boundary.

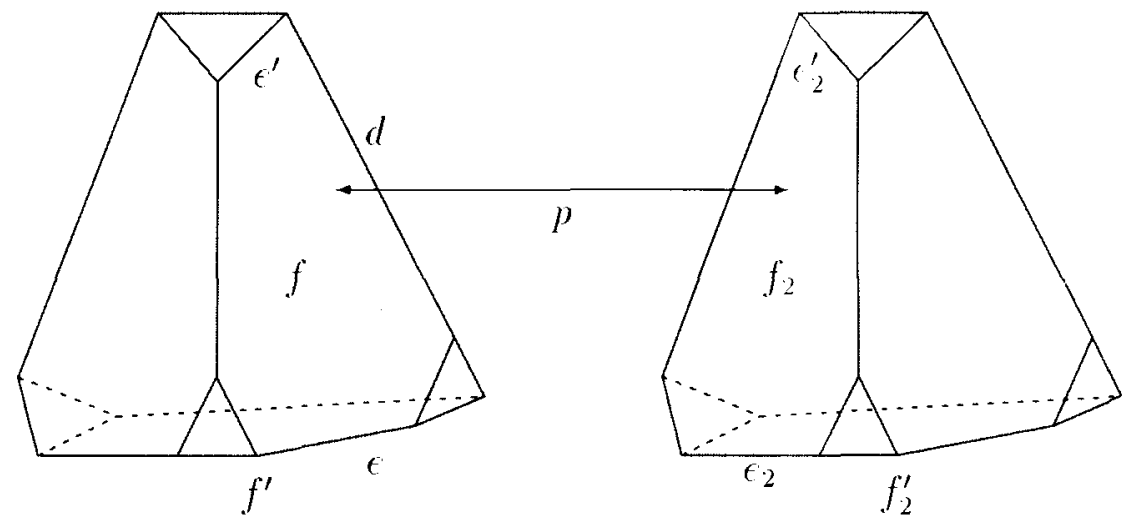

FIGURE 5. Gluing of two tetrahedra

Let $M$ be a manifold constructed out of $k$ truncated tetrahedra in this way. We consider some properties of the boundary $\partial M$ of $M$. Let $E^{\prime}$ denote the set of edges of the boundary triangles $c_{i}$ of the $k$ truncated tetrahedra. There is a natural bijection between $E$ and $E^{\prime}$, obtained by mapping $\left(e, f^{\prime}\right) \in E$ to the corresponding edge in the boundary face opposite $f^{\prime}$. For example, in Figure 5, the pair $\left(e, f^{\prime}\right)$ corresponds to the edge $e^{\prime}$. 
This bijection induces an obvious group action of $a, p$ and $c$ on $E^{\prime}$. If $e \in E^{\prime}$ is an edge of the boundary triangle $t$ then $c e$, is the next edge round clockwise in $t$. The gluing of the tetrahedra induces a corresponding gluing of the boundary triangles in which the edge $e \in E^{\prime}$ is identified with apae. In Figure 5, for example, the pair $\left(e, f^{\prime}\right)$, which corresponds to $e^{\prime}$, is mapped by $a, p$ and $a$, applied successively, to $(e, f),\left(e_{2}, f_{2}\right)$, and finally $\left(e_{2}, f_{2}^{\prime}\right)$, which corresponds to the edge $e_{2}^{\prime}$ adjoining $e$.

Since $c$ and apa map an edge in $E^{\prime}$ to, respectively, the next edge clockwise around in the same boundary triangle, and the edge with which it is identified in the neighbouring triangle, it is clear that there is a bijection between components of $\partial M$ and the orbits in $E^{\prime}$ under the action of $\langle a p a, c\rangle$. In particular, $\partial M$ is connected if and only if $\langle a p a, c\rangle$ acts transitively on $E^{\prime}$ (or equivalently on $E$ ). This condition can be simplified slightly by using a conjugate group in place of $\langle a p a, c\rangle$. We have $(c p a) a p a c(c p a)^{-1}=a p$ and $(c p a) c(c p a)^{-1}=p c a p$. Hence $\langle a p, p c\rangle=\langle a p, p c a p\rangle$ is conjugate to $\langle a p a c, c\rangle=\langle a p a, c\rangle$ so that we have

PROPOSITION 13. The boundary $\partial M$ of the manifold with boundary $M$ is connected if and only if $\langle a p, p c\rangle$ acts transitively on $E$.

The boundary $\partial M$ comprises $4 k$ boundary triangles each of which is equilateral with angles of $2 \pi / q$. The boundary, if connected, thus has Euler characteristic $\chi=4 k-6 k+12 k / q=12 k / q-2 k$. When $q=6 g(g>1)$, Theorems 7 and 9 show that $\tilde{\Gamma}^{0}(q)$ acts transitively and semiregularly on a set $S$ of $2 q=12 g$ elements, so that we may take $k=g$ in the above gluing construction to obtain a manifold $M_{g}$ with totally geodesic boundary. Corollary 10 and the above proposition then show that this boundary is connected, and so has Euler characteristic $2-2 g$ and genus $g$. We have thus shown how to construct a hyperbolic 3-manifold with totally geodesic boundary of any given genus $g \geq 2$. The volume $V_{g}$ of $M_{g}$ is $24 g$ times the volume of the prism $P(6 g)$. As $g \rightarrow \infty$ we have $V_{g} / g \rightarrow 24$ volume $P(\infty)=3.6638 \ldots$ It is known ([6]) that, for $g=2$, this construction gives a manifold with totally geodesic boundary of smallest volume, and we conjecture that for $g>2$ we obtain a least volume manifold with totally geodesic boundary of genus $g$.

If $\pi$ is a permutation of $E^{\prime}$ for which $\pi, \pi a p a$ and $\pi c$ are all semiregular of order two, then $\pi$ induces an orientation-reversing, fixed-point-free involution of $\partial M$. Hence, using Theorem 12, we can glue the genus $g$ boundary of our manifold to itself to obtain a manifold $M$ containing a non-orientable totally geodesic surface of genus $g+1$, at least for all even $g \neq 6,8$. In the other direction, given any manifold $M$ containing a non-orientable totally geodesic surface $S$, we can cut along $S$ to obtain a manifold with boundary the double cover of $S$. Consequently the least volume for a manifold with totally geodesic boundary of genus $g$ does not exceed that of a manifold containing a non-orientable totally geodesic surface of genus $g+1$. If, as we have conjectured, the manifolds that we have constructed with totally geodesic boundary 
of genus $g$ are the smallest such manifolds, we have the reverse inequality, for even $g \neq 6,8$.

\section{References}

[1] E. M. Andreev, 'On convex polyhedra in Lobačevskii spaces', Math USSR-Sb. 10 (1970), 413-440.

[2] A. F. Beardon, The geometry of discrete groups (Springer, Berlin, 1983).

[3] M. D. E. Conder and G. J. Martin, 'Cusps, triangle groups and hyperbolic 3-folds', J. Austral. Math. Soc. (Series A) 55 (1993), 149-182.

[4] H. S. M. Coxeter, 'Discrete groups generated by reflections', Ann. of Math. 2 (1934), 588-621.

[5] M. Fujii, 'Hyperbolic 3-manifolds with totally geodesic boundary which are decomposed into hyperbolic truncated tetrahedra', Tokyo J. Math. 13 (1990), 353-373.

[6] S. Kojima and Y. Miyamoto, 'The smallest hyperbolic 3-manifolds with totally geodesic boundary', J. Differential Geom. 34 (1991), 175-192.

[7] F. Lanner, 'On complexes with transitive groups of automophisms', Comm. Sém. Math. Univ. Lund. 11 (1950), 41.

[8] T. H. Marshall, PhD Thesis (University of Auckland, 1994).

[9] —_, 'Geometry of pseudospheres II', New' Zealand J. Math. to appear.

[10] B. Maskit, Kleinian groups (Springer, Berlin, 1987).

[11] J. Milnor, 'Hyperbolic geometry: the first 150 years', Bull. Amer: Math. Soc. 6 (1982), 9-24.

[12] A. Selberg, On discontinuous groups in higher-dimensional spaces (Tata Institute, Bombay, 1960).

[13] D. Singerman, 'Subgroups of Fuchsian groups and finite permutation groups', Bull. London Math. Soc. 2 (1970), 319-323.

[14] E. B. Vinberg (ed.), Geometry II, Encyclopaedia Math. Sci. 29 (Springer, Berlin, 1993).

Department of Mathematics

University of Auckland

Private Bag 92019

Auckland

New Zealand 